\title{
Ouadi el-Jarf (2020)
}

Pierre Tallet, Damien Laisney et Séverine Marchi

\section{OpenEdition \\ Journals}

Édition électronique

URL : https://journals.openedition.org/baefe/2674

DOI : $10.4000 /$ baefe. 2674

ISSN : 2732-687X

Éditeur

ResEFE

\section{Référence électronique}

Pierre Tallet, Damien Laisney et Séverine Marchi, « Ouadi el-Jarf (2020) » [notice archéologique],

Bulletin archéologique des Écoles françaises à l'étranger [En ligne], Égypte, mis en ligne le 30 mai 2021, consulté le 27 juillet 2021. URL : http://journals.openedition.org/baefe/2674 ; DOI : https://doi.org/ $10.4000 /$ baefe. 2674

Ce document a été généré automatiquement le 27 juillet 2021.

\section{cc) $(1) \odot$}

Le Bulletin archéologique des Écoles françaises à l'étranger est mise à disposition selon les termes de la Licence Creative Commons Attribution - Pas d'Utilisation Commerciale - Pas de Modification 4.0 International. 


\title{
Ouadi el-Jarf (2020)
}

\author{
Pierre Tallet, Damien Laisney et Séverine Marchi
}

\section{NOTE DE L'AUTEUR}

Année de la campagne : 2020 (7 mars - 23 mars ; 23 octobre - 25 novembre) Autorité nationale présente : Le ministère du Tourisme et des Antiquités (MoTA) était représenté par Aïd Mohamed Ahmed (Inspectorat de Suez)

Numéro et intitulé de l'opération de terrain : 17132 - Mission archéologique du ouadi el-Jarf

Composition de l'équipe de terrain : L'équipe était composée de Pierre Tallet (égyptologue, chef de mission, Sorbonne Université), Séverine Marchi (archéologue, CNRS - UMR 8167), Damien Laisney (topographe, CNRS - Maison de l'Orient et de la Méditerranée), Aurore Ciavatti (égyptologue, Ifao), Adel Farouk (intendant, MoTA), Hassan Mohamed Ahmed (restaurateur, Ifao). L'équipe de 27 ouvriers de Gourna respectant la limitation fixée à moins de trente personnes par le MoTA suite à la crise sanitaire - a été dirigée par le raïs Gamal Nasr al-Din.

Partenariats institutionnels : La mission bénéficie du soutien de Sorbonne Université / CNRS (UMR 8167 Orient et Méditerranée) et de l'université d'Assiout.

Organismes financeurs :

- Ministère de l'Europe et des Affaires étrangères

- Honor Frost Foundation

- Sorbonne Université / CNRS - UMR 8167

1 Après un lancement initial fortement perturbé au début de l'année 2020 - les opérations de terrain, engagées le 7 mars, ont dû être suspendues le 23 mars en raison de la crise sanitaire - la dixième campagne annuelle de la mission archéologique du ouadi el-Jarf a finalement pu avoir lieu cet automne, dans des conditions strictes et avec une équipe réduite au minimum, du 23 octobre au 25 novembre. La fouille s'est déroulée sur trois zones distinctes: la zone des galeries-magasins, le bâtiment intermédiaire, et la zone littorale, où des vérifications importantes ont été effectuées 
dans la perspective de la remise prochaine pour publication du premier rapport archéologique consacré au site.

\section{La zone des galeries-magasins}

2 L'intervention dans la zone des galeries G18-G28 - le deuxième système de magasins du site - s'est prolongée pendant l'ensemble de la campagne (fig. 1). Le secteur compte onze galeries, dont une double (G28), qui ont été aménagées dans le fond et en bordure d'un ouadi situé au sud du premier système de magasins G1-G17. Un récolement de l'ensemble des marques de contrôle qui ont été apposées par les équipes du début de la $I^{\mathrm{e}}$ dynastie sur les blocs de fermeture de ce second système a pu être effectué, de même qu'un point général sur l'abondante épigraphie, notamment les marques sur jarres, qui provient de ce secteur. L'ensemble démontre que ce deuxième groupe de galeries a, comme le premier, connu plusieurs occupations successives par plusieurs équipes d'ouvriers distinctes dont la présence s'échelonne probablement de la deuxième moitié du règne de Snéfrou à la fin de celui de son successeur, Chéops. Quatre des galeries, G24, G26, G28A et G28B, auront pu être au moins partiellement dégagées cette année.

Fig. 1. Plan de l'ensemble du deuxième système de galeries-magasins.

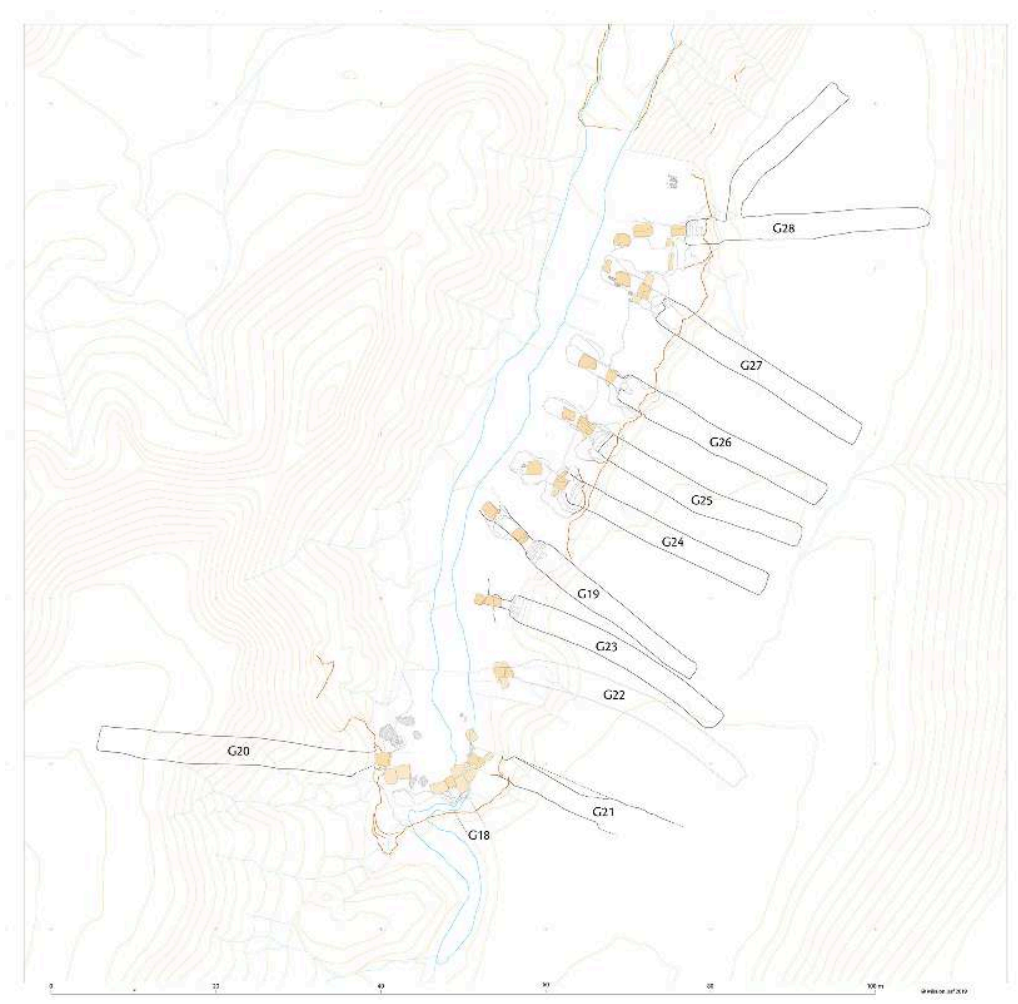

(C) Mission archéologique du ouadi el-Jarf. 17132_2020_NDMCN_001

La fouille des dix premiers mètres de la galerie G24 démontre qu'elle contient exclusivement les grosses jarres de stockage produites localement sur le site et destinées, pour l'essentiel, à assurer les réserves d'eau des missions envoyées sur ce point de la côte. Ce dépôt (fig. 2) semble proche, par sa densité, de ceux qui ont été 
observés dans les galeries mitoyennes G22 et G23. La suite du dégagement de ce magasin est programmée pour la campagne de 2021.

Fig. 2. Entrée de la galerie G24 et jarres de stockage en place.

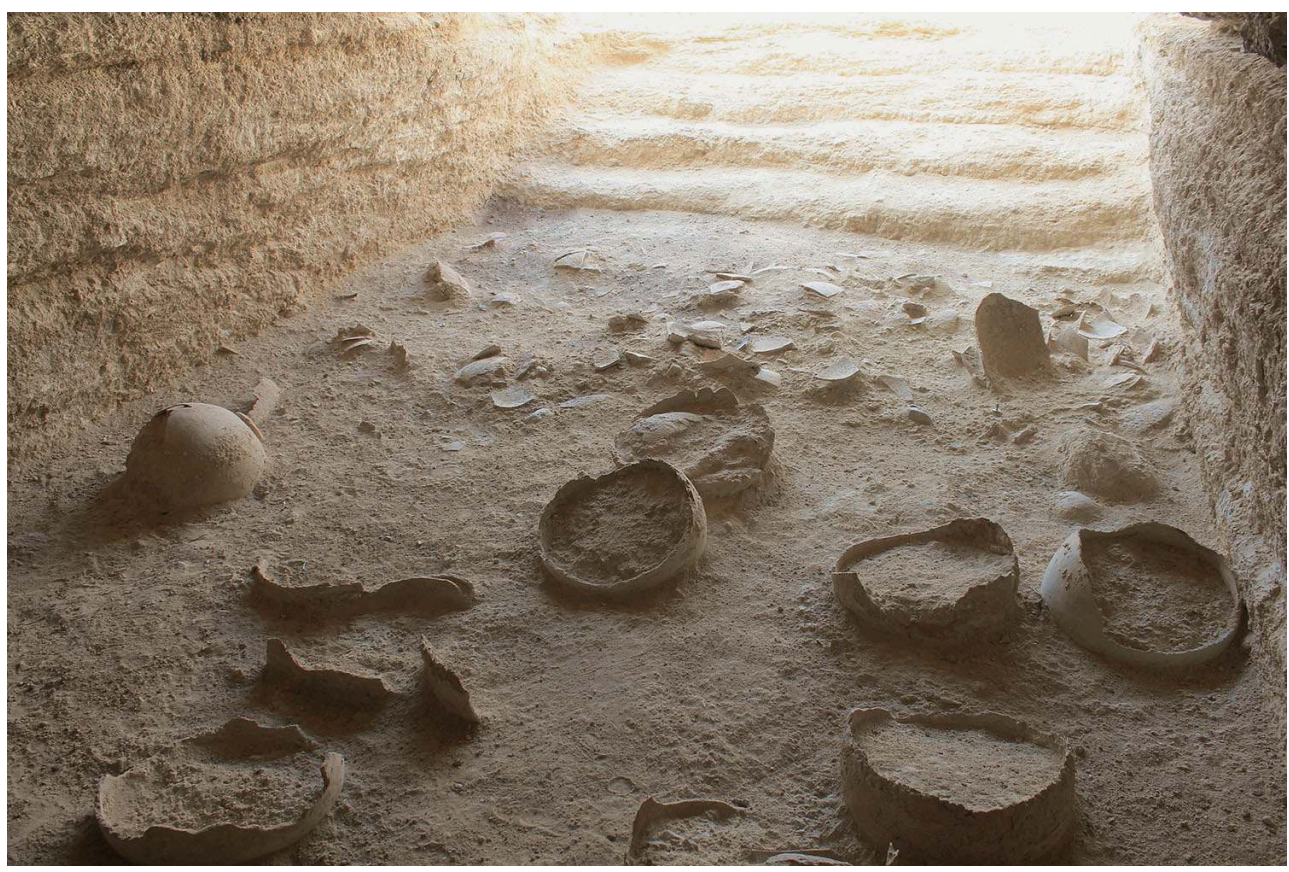

(C) Mission archéologique du ouadi el-Jarf. 17132_2020_NDMPF_001

La moitié de la galerie G26 a également été fouillée. En dépit des abondants dépôts de bois et de cordes provenant d'embarcations démontées qui avaient été recueillis lors des campagnes précédentes, qui semblaient prometteurs en mars dernier, celle-ci s'est révélée assez pauvre en matériel, seules quelques jarres brisées se trouvant au sol dans son extension. La fin de son exploration, qui n'a pas été jugée prioritaire, a donc été reportée à une campagne ultérieure, où nos moyens humains seraient moins limités que pendant la présente mission. Une partie importante du travail de dégagement a en revanche été investie dans la fouille de la galerie double G28A et G28B, qui se trouve à l'extrémité nord du ouadi où ont été installés l'ensemble de ces magasins.

La moitié de la galerie G28A - dont la fouille avait été préparée dès le mois de mars par la dépose d'une grande partie des colluvions qui la remplissent sur une hauteur de $1,50 \mathrm{~m}$ - a pu être fouillée. Elle contient elle aussi pour l'essentiel de grosses jarres de stockage de fabrication locale dans son extension. Ce matériel est mixte : il rassemble des exemplaires de plusieurs générations de ces récipients, certains marqués au nom de l'équipe $w r m_{3 j}$ "Grand est le lion» que nous pensons être la plus anciennement présente sur le site, d'autres au nom de l'équipe $m 3$ wrrt « l'uraeus <de Chéops> est <sa> Proue ", qui correspond probablement, à l'inverse, à la dernière occupation des lieux. La galerie G28B a elle aussi livré un très abondant matériel, bien plus homogène, constitué presque exclusivement de jarres brisées au sol portant la première de ces marques. Il s'agit probablement d'un dépôt ancien, qui correspond à ce qui avait été recueilli, en 2012, dans la galerie G15B. Les jarres ont manifestement servi longtemps, et portent également de très nombreuses inscriptions faites au fusain ou gravées sur la poterie (dessins, signes hiéroglyphiques) qui sont sans doute des marques de propriété. L'exploration complète de ce magasin, que nous espérons pouvoir mener à bien au 
cours de la prochaine campagne, livrera ainsi le lot le plus important d'inscriptions correspondant à cette phase présumée ancienne de l'occupation des lieux.

\section{La fouille du « bâtiment intermédiaire »}

Engagée en 2016 et poursuivie extensivement en 2017, 2018 et 2019, la fouille de la « zone 5 » a permis à la fois l'étude d'un vaste bâtiment de $57 \mathrm{~m}$ de long sur 32,50 $\mathrm{m}$ de large, probablement mis en service sous Chéops (phase II) et réoccupé dans ses niveaux d'abandon sous le règne de Chéphren (phase III), après un fort réensablement. Ce bâtiment est lui-même édifié sur les arases d'une occupation antérieure des lieux (phase I), que nous attribuons au règne de Snéfrou sur la foi de deux empreintes de sceaux fragmentaires découvertes à ce niveau. La fouille de cette phase ancienne a occupé une grande partie des campagnes de 2018 et 2019, par la dépose successive des sols de chacune des travées du bâtiment pour relever entre ses murs les structures encore visibles de cette première occupation, et en étudier le matériel. Au terme de la campagne de 2019, cette phase I avait été étudiée sur une surface de $2600 \mathrm{~m}^{2}$.

7 La campagne de 2020 avait pour objectif essentiel de dégager les installations de cette période que l'on pouvait deviner sous un talus au nord du "bâtiment intermédiaire ». Deux journées de fouille en mars 2020 avaient déjà permis de confirmer à cet endroit la présence de cellules que l'on peut rattacher à celles qui ont été fouillées dans le périmètre de la construction de l'époque de Chéops, et d'un mur d'enceinte épais, déjà observé à l'est des installations, qui semble délimiter entièrement les campements de cette première phase. La reprise du travail a permis maintenant de dégager l'ensemble de cette extrémité nord sur une distance de $60 \mathrm{~m}$ environ (fig. 3). Parmi le matériel recueilli dans ces niveaux anciens on note la présence d'un galet inscrit en rouge d'un chiffre, comparable aux « jetons » destinés à la distribution de rations alimentaires déjà découverts à différents points de ce niveau d'installation, et d'un coquillage-unio perforé pour suspension, dont l'intérieur est marqué par des traces d'encre, rouge dans la moitié gauche, noire dans la moitié droite, qui a très certainement servi de palette à encre pour un scribe assurant, à cette période, des opérations comptables dans le camp. 
Fig. 3. Vue du bâtiment intermédiaire après dégagement des structures de la phase I sur sa face nord.

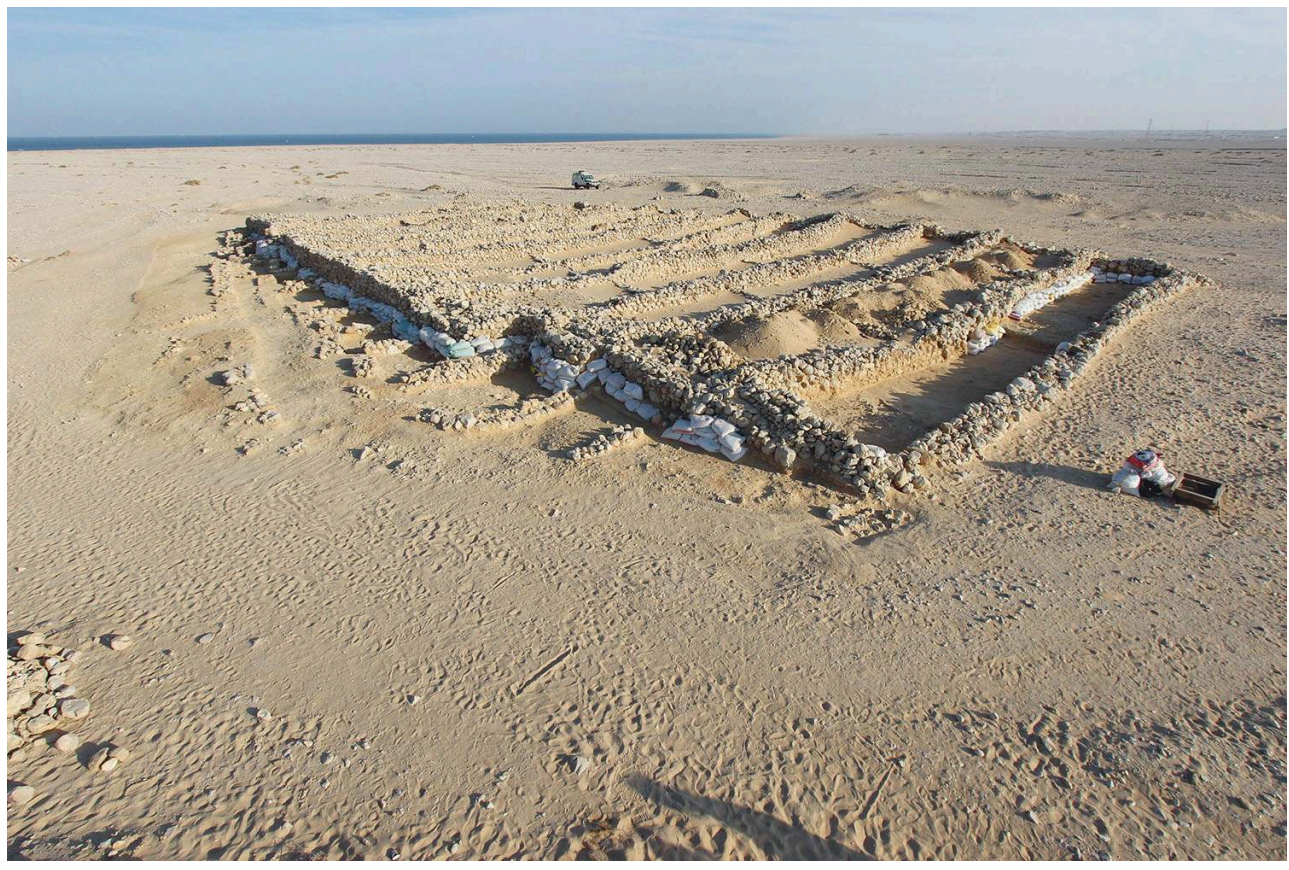

@ Mission archéologique du ouadi el-Jarf. 17132_2020_NDMPF_002

8 La fouille des sols de la travée se trouvant la plus à l'ouest (T13) a également été entreprise. Le niveau supérieur, équipé de petits murets de refend, a été déposé - des empreintes de sceaux au nom de Chéphren (phase III), qui datent cette dernière occupation, y ont été recueillies, confirmant la chronologie que nous avions déjà proposée pour les niveaux d'abandon de cette partie du site. Les niveaux correspondant à la destruction des murs de la "phase II » (état Chéops) ont ensuite été relevés. Au niveau inférieur, aucune trace évidente de la phase I n'a été observée. Contrairement à nos prévisions, il faudra malgré tout encore une campagne supplémentaire dans ce secteur pour documenter les installations de la phase I, qui s'avèrent bien plus massives, et souvent bien mieux préservées, que ce que nous imaginions au départ. Les abords ouest et sud du «bâtiment intermédiaire " devront encore être systématiquement dégagés pour pouvoir en obtenir une vision complète.

\section{La zone littorale}

Enfin, dans la perspective de la proche publication de la zone littorale du site, dont la préparation touche à son terme, nous avons dégagé à nouveau le dépôt exceptionnel de 100 ancres de bateaux rangé entre les bâtiments en peigne nord et sud de cette zone, qui avait été découvert en 2013. Cette opération était destinée à améliorer la couverture photographique qui en avait été faite lors de sa mise au jour initiale, mais elle a été également l'occasion de faire un enregistrement plus systématique des marques qui avaient été portées sur ces ancres par les équipes qui les avaient entreposées à cet endroit, en vérifiant systématiquement les faces cachées de ces objets. Le corpus de celles-ci a pu ainsi être porté, au terme de la campagne, à un ensemble de 70 documents, 32 "marques rouges", nommant pour l'essentiel les embarcations auxquelles appartenaient ces ancres, ainsi que les équipes qui leur 
étaient associées, et 38 "marques noires " - tracées pour la plupart au moyen d'un morceau de charbon - qui identifient quant à elles des phylés et des sections qui sont les subdivisions de ces mêmes équipes (fig. 4). Ce corpus nous permet ainsi d'obtenir une image de la dernière flotte de Chéops qui a fréquenté les lieux - avant la fermeture définitive de ces bâtiments du bord de mer - et de reconstituer, dans leurs grandes lignes, les arborescences de ces équipes-âper, constituées à chaque fois de quatre phylés de 40 hommes, elles-mêmes divisées en quatre sections de 10 hommes systématiquement identifiables par un symbole qui leur est associé.

Fig. 4. Ancre de bateau portant le nom de l'équipe « Doua Ouadjet» ( $a$ : photo ; b : relevé).
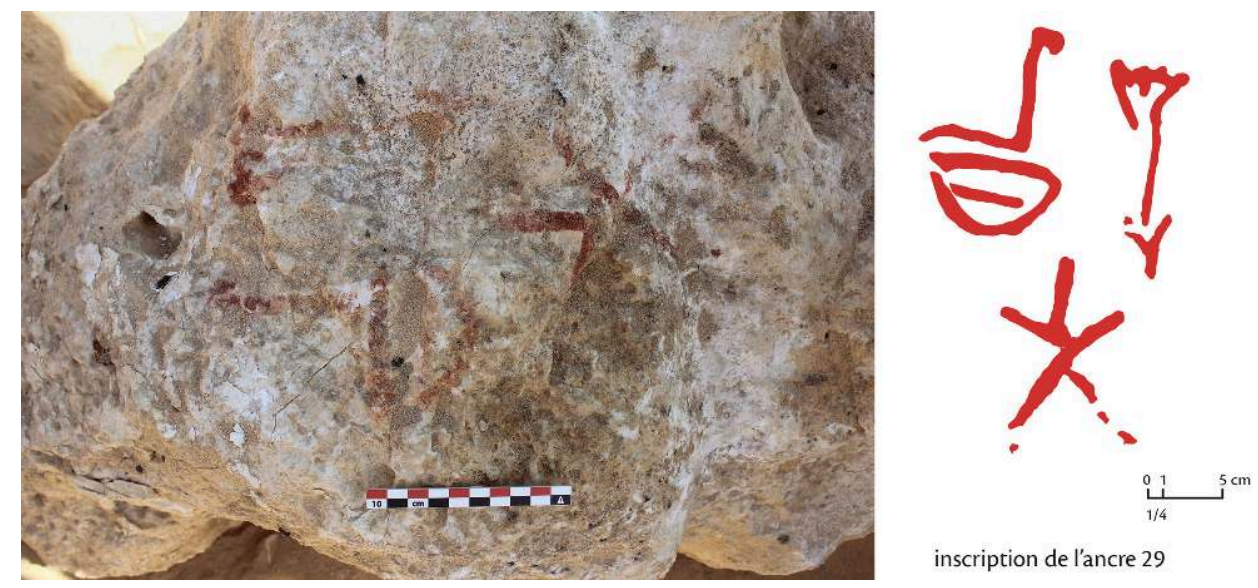

(C) Mission archéologique du ouadi el-Jarf. 17132_2020_NDMPF_003, 17132_2020_NDMDM_001

Le projet d'étude de ce port du début de la $\mathrm{IV}^{\mathrm{e}}$ dynastie a donc pu, en dépit des circonstances, enregistrer des résultats tangibles cette année, qui sont encore complétés par la poursuite de l'étude et de la restauration des papyrus découverts sur le site (un deuxième volume consacré aux «journaux de bords" qui y ont été découverts est actuellement sous presse à l'Ifao) ainsi que par l'avancement de la rédaction des deux premiers volumes consacrés à la publication du site, (Ouadi el-Jarf I. Les installations maritimes et Ouadi el-Jarf II. Le premier système de galeries-magasins) qui seront l'un comme l'autre remis pour publication dans le courant de l'année 2021. 


\section{INDEX}

\section{Thèmes : IFAO}

nature https://ark.frantiq.fr/ark:/26678/pcrtb1E0Dz7cSX

Année de l'opération : 2020

anthroponymes https://ark.frantiq.fr/ark:/26678/pcrtsK6PxtkII0, https://ark.frantiq.fr/ark:/ 26678/pcrtbZGucV9Tr9, https://ark.frantiq.fr/ark:/26678/pcrtUzj6OaUSD2

chronologie https://ark.frantiq.fr/ark:/26678/pcrtPezBqzEcKR, https://ark.frantiq.fr/ark:/ 26678/pcrtb9YobQf8Eh

lieux https://ark.frantiq.fr/ark:/26678/pcrt1ioWfobZgH

sujets https://ark.frantiq.fr/ark:/26678/pcrtwh5icnwutJ, https://ark.frantiq.fr/ark:/26678/

pcrto3fB02OhuY, https://ark.frantiq.fr/ark:/26678/pcrt6htVOpKXb1, https://ark.frantiq.fr/ark:/ 26678/pcrtqYxR5r1maz

\section{AUTEURS}

\section{PIERRE TALLET}

Égyptologue, chef de mission, Sorbonne université

\section{DAMIEN LAISNEY}

Topographe, CNRS - Maison de l'Orient et de la Méditerranée

\section{SÉVERINE MARCHI}

Archéologue, CNRS - UMR 8167 